\title{
Anquilose intencional dos caninos decíduos como reforço de ancoragem para a tração reversa da maxila. Estudo cefalométrico prospectivo
}

Omar Gabriel da Silva Filho*, Terumi Okada Ozawa*, Celeste Hiromi Okada**, Helena Yuko Okada**, Luciana Dahmen***

\section{Resumo}

Objetivo: o presente trabalho de pesquisa analisou os efeitos da tração reversa da maxila associada à anquilose intencional dos caninos decíduos superiores, mediante o emprego da cefalometria. Metodologia: o protocolo de tratamento incluiu: 1) anquilose intencional dos caninos decíduos superiores; 2) expansão rápida da maxila e 3) tração reversa da maxila, imediatamente após o término da fase ativa da expansão. A amostra foi composta de 18 crianças nos estágios de dentadura decídua e dentadura mista, com idade média inicial de 7 anos e 1 mês. O intervalo médio de tratamento com a tração reversa da maxila foi de 1 ano e 1 mês. As telerradiografias laterais foram obtidas na documentação inicial e após a correção da Classe III. Resultados e Conclusões: os resultados demonstram que os ângulos representativos da convexidade facial, NAP e ANB, aumentaram de $0^{\circ}$ para $6,6^{\circ}$ e $3,5^{\circ}$, respectivamente. Isso significa dizer que a face transformou-se de reta ou côncava, peculiar na Classe III, para uma face convexa, característica de normalidade no estágio avaliado. Essa melhora na convexidade facial é atribuída ao avanço da maxila, registrado tanto na região alveolar (ângulo SNA e as distâncias Co-A e NPerp-A) como na região basal (ângulo SN.ENA). A maxila deslocou-se para frente, enquanto a redução do ângulo SNB de $80,56^{\circ}$ para $79,61^{\circ}$ demonstrou um retroposicionamento mandibular. Além da mudança no sentido sagital, houve rotação da mandíbula no sentido horário, com aumento dos ângulos SN.GoGn e SN.Gn. Somado aos efeitos ortopédicos, houve inclinação vestibular dos incisivos superiores.

Palavras-chave: Má oclusão de Classe III de Angle. Maxila. Cefalometria.

* Ortodontista do Hospital de Reabilitação de Anomalias Craniofaciais da Universidade de São Paulo (HRAC-USP), Bauru-SP.

** Professora do Curso de Ortodontia Preventiva e Interceptiva da Sociedade de Promoção Social do Fissurado Lábio-Palatal - PROFIS.

*** Aluna do Curso de Ortodontia Preventiva e Interceptiva da Sociedade de Promoção Social do Fissurado Lábio-Palatal - PROFIS. 


\section{INTRODUÇÃO}

Desde a divulgação da máscara facial por Delaire $^{8}$, o emprego da tração reversa da maxila como forma de tratamento da má oclusão de Classe III é quase convenção, tamanha a freqüência com que é usada e publicada na literatura ${ }^{1,2,6,7,9,11,13,14,15,17,19,20,22,23,24,27,28,29,30,31,32,33,34,35}$, 37,38,39,40,42,43 (Quadro I). A pretensão primeira de tal abordagem é ortopédica e consiste em estimular o crescimento sutural da face média para corrigir a discrepância sagital com o avanço da maxila. A comprovação de sua possibilidade vem dos estudos com animais. Os efeitos provocados pela máscara facial em macacos demonstram avanço da maxila com abertura e remodelação das suturas circumaxilares. Isto implica em estiramento das fibras do tecido conjuntivo, remodelação das suturas e neoformação óssea ${ }^{10,17,18,26,27}$. Os modelos fotoelásticos e biomecânicos tornam compreensíveis os efeitos mecânicos imediatos induzidos pela tração reversa da maxila ${ }^{25}$.

A circunstância da tração reversa da maxila ser ancorada no arco dentário superior, portanto, longe do centro de resistência da maxila, compromete os propósitos da Ortopedia de avanço maxilar e justifica o grau variável, mas importante, de compensação dentária. Para potencializar a ancoragem, a expansão rápida da maxila usando o aparelho expansor fixo com parafuso ${ }^{14,22,23,42}$ tem sido usada associada à tração reversa da maxila, com o intuito de: 1) romper a sutura palatina mediana durante o acionamento do parafuso expansor, imediatamente antes da tração reversa; 2) unir dentes e base óssea numa unidade, sobretudo quando o aparelho é dento-muco-suportado e 3) estabelecer pontos de inserção intrabucais para a ação ortopédica sagital; tudo isso visa potencializar o efeito ortopédico durante a tração reversa da maxila ${ }^{14}$. $\mathrm{O}$ efeito ortopédico induzido pela tração reversa da maxila é mais significativo quando a expansão rápida da maxila antecede o uso da máscara facial ${ }^{2}$, com conseqüente redução da compensação dentária ${ }^{21}$.
A literatura e a prática têm mostrado que a Ortopedia mecânica usada para avanço da maxila induz efeitos ortopédicos e ortodônticos que melhoram em graus variados a oclusão e a estética facial. Esses efeitos poderiam ser resumidos em: 1 ) deslocamento

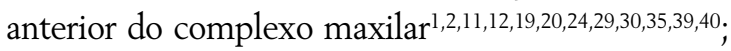
2) deslocamento anterior dos dentes superiores $^{3,12,16,20,35,41}$; 3) rotação da mandíbula para baixo e para trás ${ }^{11,20,35}$ e 4) inclinação dos incisivos inferiores para lingual ${ }^{2,6,11,19,30,35}$. Os efeitos ortopédicos são mais expressivos em estágios mais precoces do desenvolvimento da oclusão, com avanços significativos dos pontos A, ENA e ENP1,19,31,38,40.

A anquilose intencional de caninos decíduos tem sido usada com o propósito de potencializar os efeitos ortopédicos e reduzir os efeitos ortodônticos da tração reversa da maxila ${ }^{34}$.

O objetivo do presente estudo consistiu em avaliar os efeitos ortodônticos e ortopédicos imediatos induzidos por um protocolo de tratamento ortopédico do Padrão esquelético III nos estágios de dentadura decídua e mista, que inclui: anquilose intencional dos caninos decíduos, aparelho expansor fixo dento-muco-suportado (tipo Haas) e tração reversa da maxila com máscara facial.

\section{MATERIAL E MÉTODOS}

A casuística do presente trabalho consistiu de 18 crianças, na faixa etária compreendida entre 5 anos e 8 anos e 11 meses, sendo 8 do gênero feminino e 10 do gênero masculino, regularmente matriculadas no curso de Ortodontia Preventiva e Interceptiva da PROFIS - Bauru. Todas elas se encontravam no estágio de dentadura decídua ou mista e apresentavam Padrão esquelético III, com mordida cruzada anterior ou de topo acompanhada ou não de mordida cruzada posterior.

O protocolo de tratamento obedeceu os seguintes passos operacionais: 1) anquilose intencional dos caninos decíduos superiores (procedimento já descrito em artigo prévio ${ }^{34}$ ); 2) ativação do aparelho expansor fixo tipo Haas, na velocidade de uma volta completa por dia, até a ruptura da su- 
SILVA FILHO, 0. G.; OZAWA, T. 0.; OKADA, C. H.; OKADA, H. Y.; DAHMEN, L.

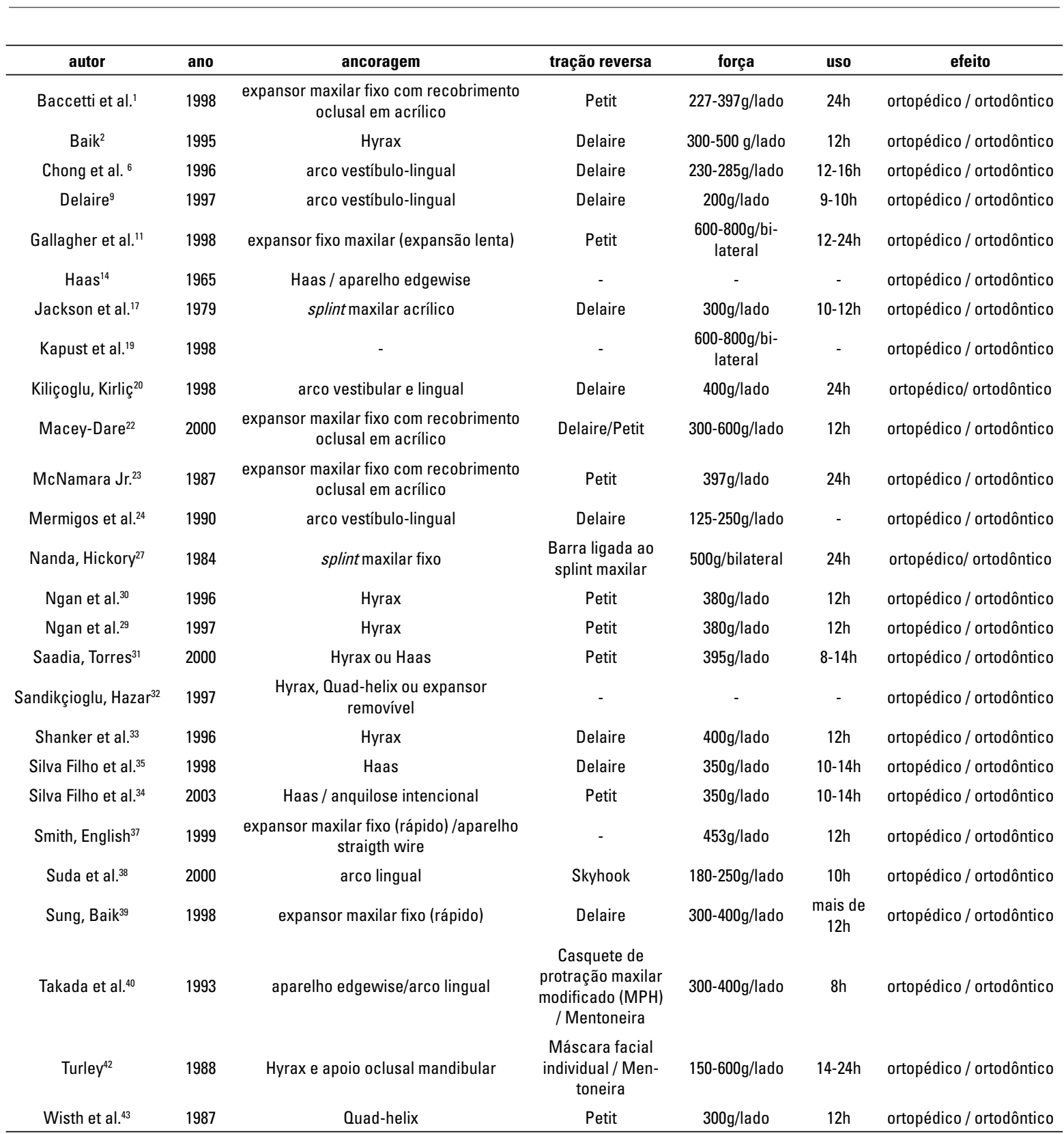

Quadro 1 - Compilação da literatura pertinente ao uso da tração reversa da maxila.

tura palatina mediana (4 voltas completas) ou até o descruzamento da mordida cruzada posterior; 3) tração reversa da maxila com máscara facial tipo Delaire, a partir de ganchos soldados na região dos caninos decíduos. O período de uso diário da máscara facial foi de 14 horas, até completar um ano ou até a esfoliação dos caninos decíduos anquilosados.

Duas telerradiografias foram usadas para avaliar os efeitos da tração reversa da maxila, uma obtida na documentação ortodôntica inicial e outra ao final da tração reversa da maxila. As seguintes grandezas cefalométricas representadas nas figuras 
1 a 4 foram empregadas: grandezas esqueléticas sagitais angulares (SN.ENA, SNA, SNB, ANB, NAP); grandezas esqueléticas lineares (N-PerpA, N PerpPog, Co-A, Co-Gn, AFAI); grandezas esqueléticas verticais angulares (SN.PO, SN.GoGn, SN.Gn) e grandezas dentárias angulares (1.PP, IMPA).

\section{RESULTADOS}

A tabela 1 exibe as estatísticas descritivas com os valores médios e os desvios-Padrão para as grandezas pré-tratamento e pós-tratamento, bem como a diferença entre os valores pré e pós-tratamento. Os testes $t$ pareados foram aplicados para verificar se existiam diferenças entre os resultados iniciais e finais. Para as grandezas representativas do comportamento dentário (IMPA e 1.PP) foram aplicados os testes $t$ não pareados, pois em algumas crianças não foi possível mensurar essas medidas na telerradiografia inicial, pela ausência dos incisivos permanentes em oclusão.

\section{DISCUSSÃO}

O tratamento ortopédico precoce da Classe III associada ao Padrão III é, sem dúvida, bastante complexo, pensando-se em termos de estabilidade, pelo fato do resultado em longo prazo não refletir necessariamente o resultado imediato. $\mathrm{O}$ prognóstico difere da Classe II com Padrão II, onde os efeitos ortodônticos e ortopédicos conseguidos com a ancoragem extrabucal ${ }^{4}$ mostram uma certa estabilidade. Não se pode ignorar que o mais fundamental para o comportamento da oclusão e da face após o tratamento de uma má oclusão Classe III é o potencial de crescimento facial. A mudança esquelética e principalmente a compensação dentária conseguidas com a Ortopedia Mecânica têm chance de serem preservadas ao longo dos anos somente se o crescimento facial permitir, o que significa dizer se o crescimento facial manifestar-se como Padrão I. Se o crescimento facial comportar-se como Padrão III, o tratamento precoce estará comprometido. Isso implica necessariamente em um tempo prolongado de contenção e acompanhamen- to, com a possibilidade de ser necessário desfazer as compensações adquiridas, com vistas à cirurgia ortognática na maturidade esquelética ${ }^{5}$. Independentemente da influência que o crescimento facial exerce na estabilidade pós-tratamento, o que se pretende de imediato, em um tratamento ortopédico para o Padrão III, é o máximo de mudanças esqueléticas com o mínimo de compensação dentária.

O presente estudo avaliou os efeitos imediatos da tração reversa da maxila, usando como ancoragem a anquilose intencional dos caninos decíduos, associada ao aparelho expansor fixo tipo Haas, para o tratamento da má oclusão Classe III. Essa abordagem ortopédica, representada na figura 5 , trata o Padrão III na maxila, já que esta encontra-se deficiente em grande parte dos indivíduos com esse Padrão III ${ }^{36}$. As crianças encontravam-se no estágio de dentadura decídua e mista precoce, já que a integridade radicular dos caninos decíduos é exigência primeira para a realização da anquilose intencional desses dentes. $\mathrm{O}$ uso médio da máscara facial foi de 1 ano e 1 mês e nenhuma das crianças exibiu incômodo que obrigasse a suspensão antecipada do tratamento. A presente pesquisa não faz análise comparativa entre a ancoragem convencional (tração reversa da maxila ancorada no aparelho expansor fixo tipo Haas) e a ancoragem reforçada com a ruptura do ligamento periodontal (anquilose intencional dos caninos decíduos). Comparar o modelo ortopédico convencional com a adição da anquilose

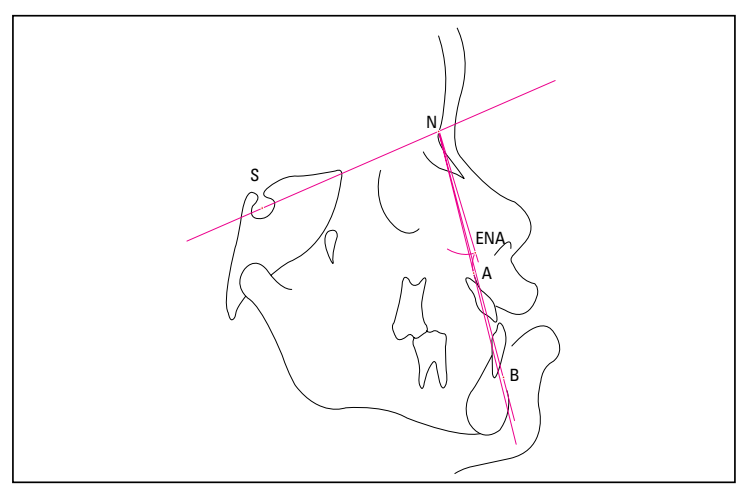

FIGURA 1 - Traçado cefalométrico com as grandezas esqueléticas sagitais angulares: SN.ENA, SNA, SNB, ANB. 


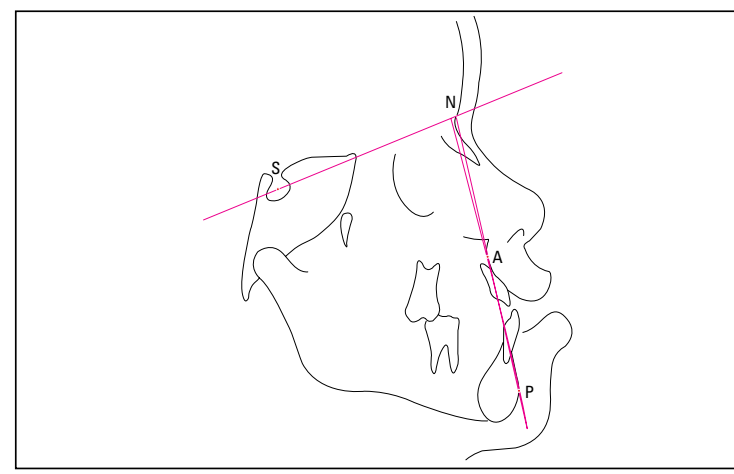

FIGURA 2 - Traçado cefalométrico com a grandeza esquelética sagital NAP.

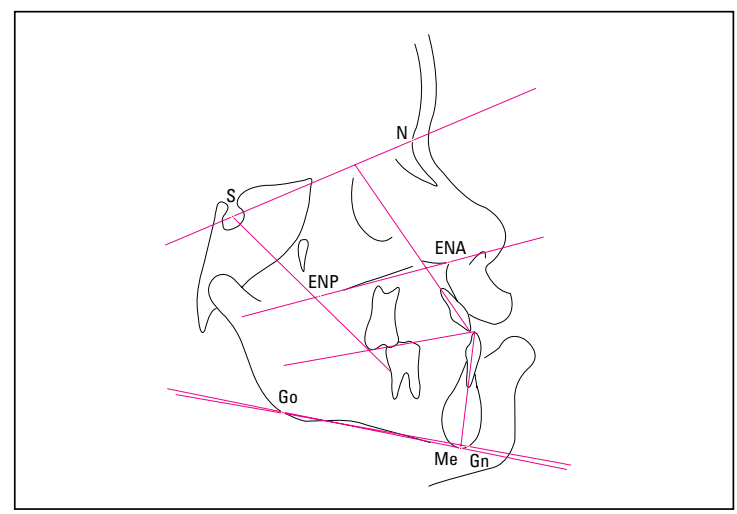

FIGURA 4 - Traçado cefalométrico com as grandezas esqueléticas dentárias: SN.PO, SN.GoGn, SN.Gn, 1.PP, IMPA.

intencional dos caninos decíduos constitui proposta futura. No entanto, levando-se em consideração os dados de pesquisa prévia da mesma instituição, envolvendo tração reversa da maxila ancorada no aparelho expansor fixo tipo $\mathrm{Haas}^{35}$, pode-se deduzir que a ancoragem biológica potencializa o efeito esquelético nas maxilas. Pelo menos isso é o que sugerem os valores referentes às medidas cefalométricas SNA, ANB, NAP, N-PerpA na abordagem sem anquilose intencional dos caninos decíduos (Tab. 2), quando comparados com os dados da tabela 1 .

$\mathrm{Na}$ presente amostra, a maxila deslocou-se para frente durante o período de tratamento (Tab. 1). De fato, esse é o efeito específico e singular esperado pelo emprego da tração reversa da maxila. Essa supremacia da Ortopedia Maxilar, pela rapidez com que promove o avanço da face média, em re-

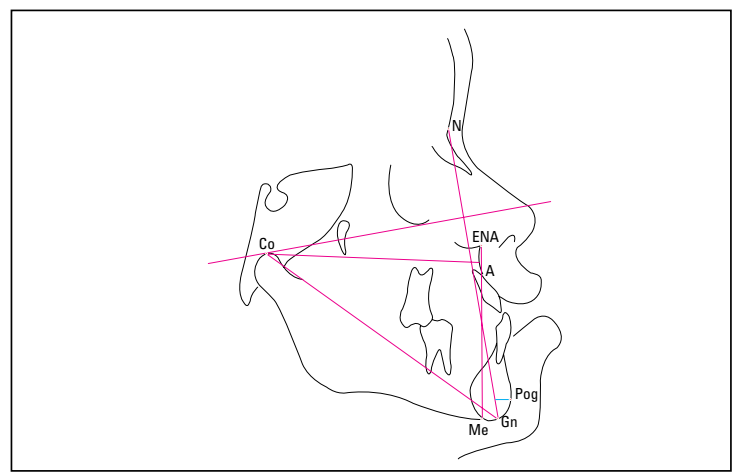

FIGURA 3 - Traçado cefalométrico com as grandezas esqueléticas: Nperp-A, Nperp-Pog, Co-A, Co-Gn, AFAl.

Tabela 1 - Comportamento das bases apicais e dos arcos dentários, conseqüente ao avanço ortopédico das maxilas com máscara facial, usando como ancoragem a anquilose intencional dos caninos decíduos e o aparelho expansor fixo tipo Haas.

\begin{tabular}{|c|c|c|c|c|c|c|}
\hline & \multicolumn{2}{|c|}{ inicial } & \multicolumn{2}{|c|}{ final } & \multirow{2}{*}{$\begin{array}{c}\text { dife- } \\
\text { rença }\end{array}$} & \multirow{2}{*}{ teste $t$} \\
\hline & média & d.p. & média & d.p. & & \\
\hline \multicolumn{7}{|c|}{ grandezas esqueléticas sagitais angulares } \\
\hline NAP & $-0,11$ & 7,44 & 6,64 & 7,31 & 6,75 & $<0,001^{*}$ \\
\hline SNA & 80,64 & 2,95 & 83,11 & 4 & 2,47 & $<0,001^{*}$ \\
\hline SNB & 80,56 & 3,93 & 79,61 & 3,47 & $-0,95$ & 0,056 \\
\hline ANB & 0,08 & 3,21 & 3,5 & 3,5 & 3,42 & $<0,001^{*}$ \\
\hline SN.ENA & 84,17 & 4,05 & 86,97 & 4,64 & 2,8 & $<0,001^{*}$ \\
\hline \multicolumn{7}{|c|}{ grandezas esqueléticas sagitais lineares } \\
\hline Nperp-A & $-1,78$ & 2,32 & 0,81 & 3,06 & 2,59 & $<0,001^{*}$ \\
\hline Nperp-Pog & $-2,19$ & 6,74 & $-3,78$ & 5,05 & 1,59 & 0,172 \\
\hline Co-A & 77,28 & 3,89 & 81,81 & 4,41 & 4,53 & $<0,001^{*}$ \\
\hline Co-Gn & 102,69 & 5,86 & 105,42 & 6,24 & 2,73 & 0,013 \\
\hline AFAl & 59,22 & 4,77 & 62,17 & 5,58 & 2,95 & $<0,001^{*}$ \\
\hline \multicolumn{7}{|c|}{ grandezas esqueléticas verticais angulares } \\
\hline SN.PO & 19,36 & 6,21 & 18,67 & 5,91 & 0,69 & 0,751 \\
\hline SN.GoGn & 35 & 6,62 & 36,83 & 6,26 & 1,83 & $0,008^{*}$ \\
\hline SN.Gn & 65,69 & 5,07 & 67,36 & 4,45 & 1,67 & $<0,001^{*}$ \\
\hline \multicolumn{7}{|c|}{ grandezas dentárias angulares } \\
\hline 1.PP & 107,08 & 9,21 & 110,85 & 6,27 & 3,77 & 0,251 \\
\hline IMPA & 83,17 & 6,29 & 83,88 & 6,44 & 0,71 & 0,768 \\
\hline
\end{tabular}

${ }^{*}$ Estatisticamente significante (com nível de significância de 1\%)

lação à mentoneira, explica-se provavelmente por não ir contra o fluxo espontâneo do crescimento maxilar. $\mathrm{Na}$ faixa etária aqui estudada, dentadura decídua e início da dentadura mista, o deslocamento do ponto A para frente tende a manifestar-se em cerca de $90 \%$ dos pacientes ${ }^{9}$, o que incentiva o uso dessa abordagem terapêutica. A tabela 1 mostra 
que os ângulos SN.ENA e SNA aumentaram cerca de $2,5^{\circ}$, refletindo o deslocamento da base óssea e do alvéolo, respectivamente. $\mathrm{O}$ único trabalho onde o aumento do ângulo SNA superou o do presente artigo foi o de Göyenç e Ersoy ${ }^{12}$, onde o valor do SNA aumentou cerca de $3^{\circ}$. Kapust et al. ${ }^{19}$, Kiliçoglu e Kirliç ${ }^{20}$ registraram a mesma magnitude de comportamento para o ângulo SNA. Mermigos et al. ${ }^{24}$ constataram um aumento de $1,7^{\circ}$. Sung e Baik $^{39}$ assinalaram aumentos para o ângulo SNA entre $1^{\circ}$ e $2,1^{\circ}$, na dependência da faixa etária. Chong et al. ${ }^{6}$ e Gallagher et al. ${ }^{11}$ também observaram comportamento idêntico, porém em menor magnitude, próximo de $1^{\circ}$, ou entre $0,73^{\circ}$ e $1,57^{\circ} 31$, sem significância quando comparado com um grupo controle $e^{6}$.

O aumento do ângulo SNA é conseqüência do deslocamento do ponto $\mathrm{A}$ para frente. $\mathrm{O}$ avanço do ponto A foi menor quando a maxila foi tracionada sem o uso da anquilose intencional dos caninos decíduos. A comparação com a tabela 2 sugere que o deslocamento do ponto A duplicou com a ancoragem biológica. As grandezas cefalométricas lineares NPerp-A e Co-A reafirmam o comportamento do ponto $\mathrm{A}$, ao mostrarem aumentos significativos, de $2,5 \mathrm{~mm}$ e $4,5 \mathrm{~mm}$, respectivamente, principalmente levando-se em consideração o pouco tempo de acompanhamento, por volta de 1 ano. Esses valores ratificam o deslocamento do ponto A induzido pela tração reversa da maxila. A literatura tem confirmado o deslocamento anterior linear do ponto $\mathrm{A}$, com avanço de 1 a $2 \mathrm{~mm}^{11,23}, 1,76 \mathrm{~mm}^{24}, 2 \mathrm{~mm}^{30}$, $2,2 \mathrm{~mm}^{40}, 2,3 \mathrm{~mm}^{19}, 2,4 \mathrm{~mm}^{16,33}, 2,5 \mathrm{~mm}^{35}, 3 \mathrm{~mm}^{20} \mathrm{e}$ variações de $1,7 \mathrm{~mm}$ a $2,8 \mathrm{~mm}$, em faixas etárias que oscilarem entre 7 e 12 anos $^{39}$. Esse deslocamento reflete o aumento do comprimento do corpo maxilar. Sung e Baik ${ }^{39}$ mediram o comprimento do corpo maxilar entre os pontos ENA e ENP e chegaram à conclusão que a tração reversa da maxila induz aumento do comprimento maxilar, em comparação com um grupo controle sem tratamento. $\mathrm{O}$ aumento no comprimento maxilar no grupo com tração reversa da maxila variou de $1,2 \mathrm{~mm}$ a $1,5 \mathrm{~mm}$, em comparação com o grupo controle de $0,5 \mathrm{~mm}$ a $0,9 \mathrm{~mm}$. Mermigos et al. ${ }^{24}$ relataram um aumento do comprimento maxilar de $2,4 \mathrm{~mm}$.

O ângulo SNB mostrou uma redução aproximada de $1^{\circ}$, sem significância estatística (Tab. 1). Essa redução pode ser reflexo geométrico da rotação da mandíbula no sentido horário, já relatada na literatura ${ }^{2,31}$ e comprovada pelo aumento estatisticamente significante dos ângulos SN.GoGn e SN.Gn e pela ausência de significância na medida Nperp-Pog. Kapust et al. ${ }^{19}$, Sung e Baik ${ }^{39}$ observaram uma redução média do ângulo SNB em torno de $1,5^{\circ}$. Chong et al. ${ }^{6}$ não encontraram alteração no ângulo SNB. Mas, quando comparado com um grupo controle sem tratamento, encontraram um menor comprimento do corpo mandibular no grupo tratado. Bacetti et al. ${ }^{1}$ encontraram incrementos

Tabela 2 - Comportamento sagital dos maxilares conseqüente ao emprego da expansão rápida e tração reversa da maxila ${ }^{35}$.

\begin{tabular}{ccccccc}
\hline & \multicolumn{2}{c}{ inicial } & \multicolumn{2}{c}{ final } & diferença & teste t \\
\cline { 2 - 5 } & $\mathbf{x}$ & d.p. & $\mathbf{x}$ & d.p. & & \\
\hline SNA & 82 & 3,65 & 83 & 4,05 & 1,09 & $*$ \\
SNB & 80,68 & 3,49 & 79,6 & 3,5 & -1 & $*$ \\
ANB & 1,42 & 1,74 & 3,48 & 1,81 & 2,06 & $*$ \\
NAP & 2,61 & 4,57 & 6,76 & 4,29 & 4,14 & $*$ \\
N-PerpA & $-0,71$ & 2,96 & 0,77 & 3,79 & 1,48 & $*$ \\
\hline
\end{tabular}

* Estatisticamente significante (com nível de significância de 1\%).
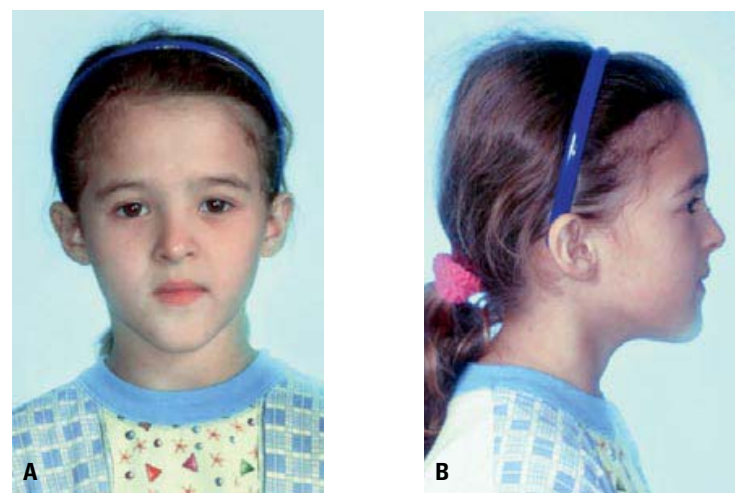

FIGURA 5 - A) Padrão dolicofacial, B) com deficiência maxilar. 

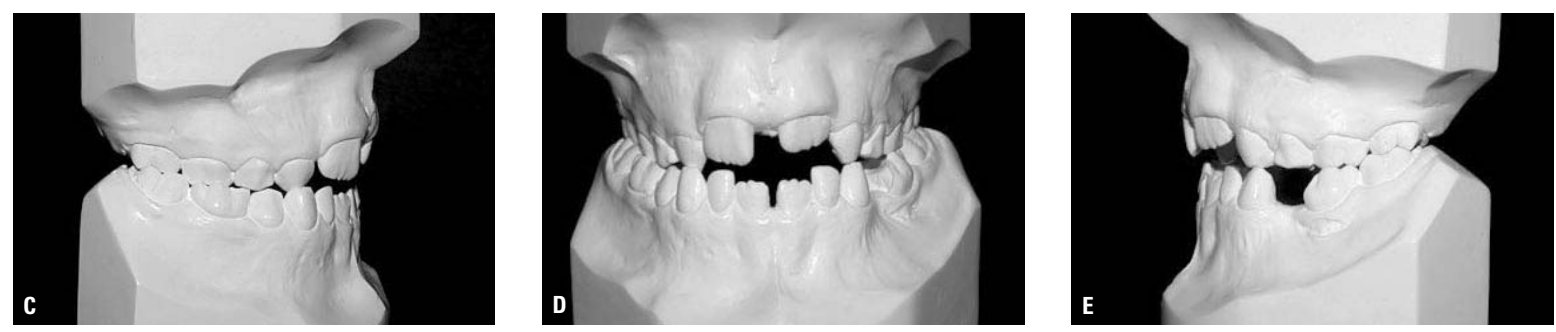

FIGURA 5 - C, D, E) Deficiência maxilar com apinhamento ântero-superior.
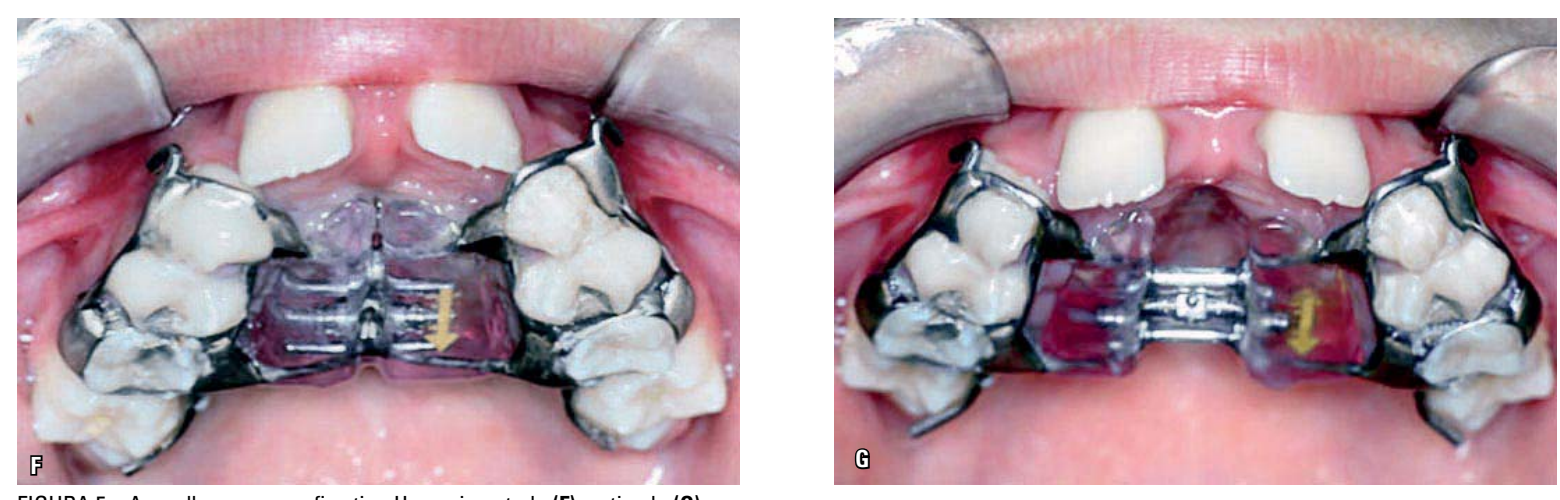

FIGURA 5 - Aparelho expansor fixo tipo Haas cimentado (F) e ativado (G).
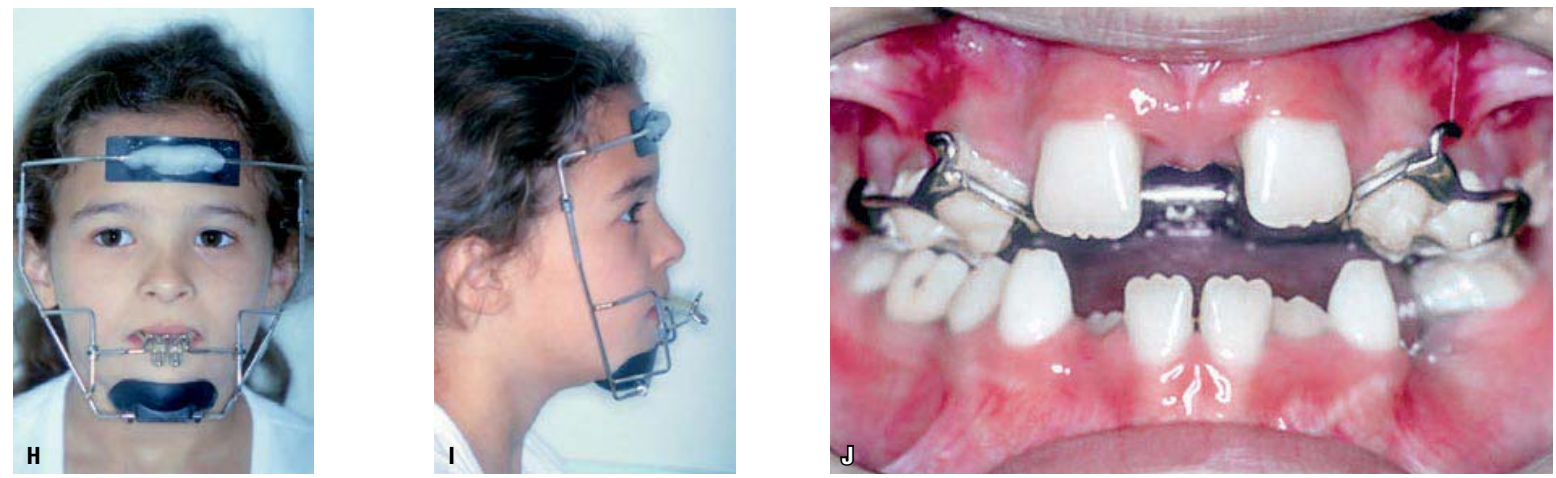

FIGURA 5 - Tração reversa da maxila mediante o uso da máscara facial (H, I) ancorada no aparelho expansor fixo tipo Haas (J).

anualizados significantemente menores de crescimento mandibular nos pacientes tratados com a máscara facial no início da dentadura mista, em comparação com um grupo controle. Esses resultados levantam a polêmica se a reação da máscara facial é suficiente para influenciar o crescimento mandibular.

Os ângulos de convexidade, NAP e ANB, sofreram impacto significativo com a mecânica ortopédica de tração reversa da maxila e isso é importante para delinear uma nova face ao paciente. A repercussão clínica dessas mudanças na face é importante, pois reflete na estética facial. As mudanças angulares foram de $6,64^{\circ}$ e $3,5^{\circ}$, para os ângulos NAP e ANB, respectivamente, maiores que os $4,14^{\circ}$ e $2,06^{\circ}$ registrados em trabalho prévio sem a anquilose intencional dos caninos decíduos ${ }^{35}$, como se observa na tabela 2. 


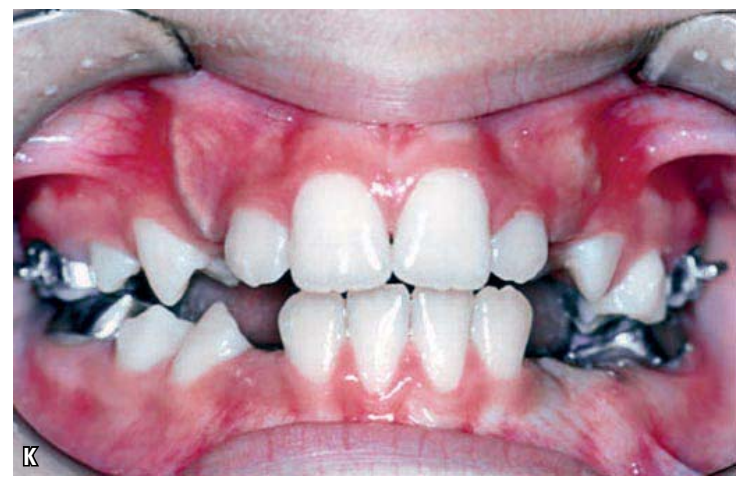

FIGURA 5 - Oclusão (K) e face (L, M) após a abordagem ortopédica.
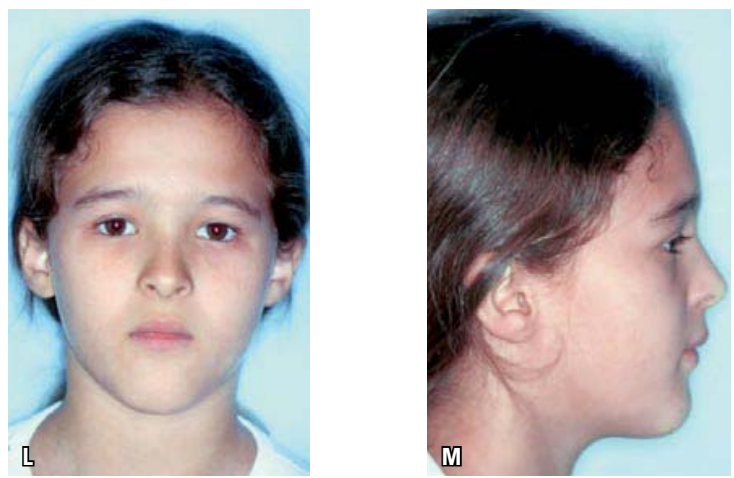
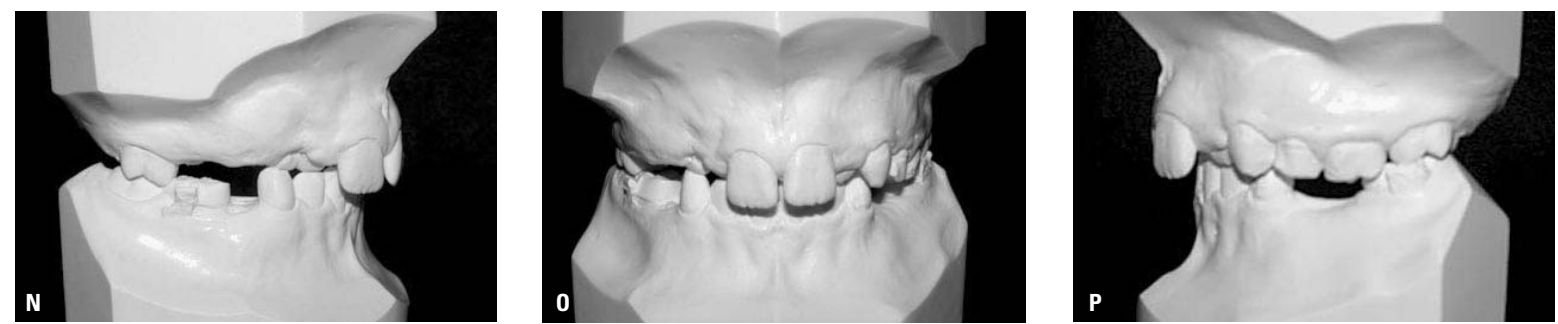

FIGURA 5 - Modelos após a remoção do aparelho expansor fixo tipo Haas e após a suspensão da máscara facial (N, $\mathbf{0}, \mathbf{P})$.

Chong et al. ${ }^{6}$ e Kiliçoglu e Kirliç ${ }^{20}$ conseguiram aumentos de apenas $2^{\circ}$ e $4,34^{\circ}$ no ângulo ANB. No entanto, esses autores não usaram aparelho expansor como ancoragem. $\mathrm{O}$ arco palatino substituiu o aparelho expansor em crianças de 4,5 a 8,2 anos de idade, com um tempo médio de uso da máscara de 12 meses.

Os dois ângulos que registram o comportamento vertical da mandibula (SN.GoGN e SN.Gn) denunciam rotação mandibular no sentido horário, resultado semelhante ao registrado em trabalhos prévios $^{35,40,43}$. Chong et al. ${ }^{6}$ mencionaram que o principal responsável pela melhora na relação sagital entre as bases apicais foi essa rotação mandibular para baixo e para trás. A rotação horária da mandíbula contribuiu também para o aumento na altura facial ânterior-inferior (AFAI) de 2,9mm.

A angulação do plano oclusal (SN.PO) mudou pouco durante a tração reversa da maxila, um pouco mais que $0,5^{\circ}$. Essa mudança estatisticamente insignificante deve-se ao ponto de inserção do elástico de tracionamento, na região dos caninos, diretamente no aparelho expansor fixo tipo Haas. A tração maxilar a partir da região posterior favorece o abaixamento da parte posterior das maxilas ${ }^{16}$, enquanto a tração a partir dos caninos tende a reduzir esse efeito rotacional durante a protração maxilar ${ }^{17,25,26,39}$.

Uma das pretensas intenções da anquilose intencional dos caninos decíduos é eliminar a compensação dentária superior. Não que a compensação dentária terapêutica seja intolerável, mesmo porque é inevitável em um tratamento ortopédico. No entanto ela deve estar dentro do limite de aceitação periodontal. Como não se consegue eliminar por completo a compensação dentária, uma ancoragem reforçada poderia reduzir a participação dentoalveolar na tração reversa da maxila. Os efeitos dentoalveolares no presente estudo foram avaliados mediante o emprego de duas grandezas: os ângulos 1.PP e IMPA. O comportamento desses ângulos expressa algo instigante, ou seja, os incisivos superiores inclinaram para vestibular cerca 
de $3,5^{\circ}$ e os incisivos inferiores mantiveram suas posições iniciais. Esses dados discordam dos de Chong et al. ${ }^{6}$, Kapust et al. ${ }^{19}$, Silva Filho et al. ${ }^{35}$ e Wisth et al. ${ }^{43}$, os quais encontraram compensação apenas no arco dentário inferior. O comportamento do ângulo 1.PP sublinha a propensão para a compensação dentária superior nos pacientes com anquilose intencional de caninos decíduos. Esse resultado deixa claro que a anquilose intencional dos caninos decíduos pode até potencializar o efeito ortopédico induzido pela tração reversa da maxila, mas não evita a compensação dentária superior, representada pela vestibularização dos incisivos superiores.

\title{
Intentional ankylosis of the deciduous canines to enhance maxillary protraction. A prospective cephalometric analysis
}

\begin{abstract}
Aim: the current article analyses the effects of maxillary protraction associated to the intentional ankylosis of the deciduous canines on the basis of cephalometric measurements. Methods: the treatment protocol included: 1) intentional ankylosis of the upper deciduous canines; 2) rapid palatal expansion and 3) maxillary protraction performed immediately after the end of the expansion. The sample was comprised of 18 patients equally divided according to gender, in the primary and mixed dentition. The mean treatment time with maxillary protraction was 1 year. The lateral radiographs were taken in the beginning of the treatment and after correction of the Class III malocclusion. Results and Conclusion: the results show that the facial convexity angles - NAP and ANB - increased from $0^{\circ}$ to $6.6^{\circ}$ and $3.5^{\circ}$, respectively. This means that the patients' profile changed from straight or concave, which is typical in the Class III malocclusion, to convex, which is typical in normal occlusion. Such an improvement in the facial convexity is due to the maxillary advancement, evidenced both in the dentoalveolar (SNA angle and Nperp-A measurement) and in the basal areas (SN.ANS angle). The maxilla was anteriorly displaced while the reduction of the SNB angle from $80.56^{\circ}$ to $79.61^{\circ}$ demonstrated a mandibular retropositioning. Besides the sagittal alterations, the mandible presented a clockwise rotation, with increase in the SN.GoGn and SN.Gn angles. Dental compensation was also noticed, represented by the buccal inclination of the upper incisors.
\end{abstract}

Key words: Angle Class III malocclusion. Maxilla. Cephalometry.

\section{REFERÊNCIAS}

1. BACCETTI, T. et al. Skeletal effects of early treatment of Class III malocclusion with maxillary expansion and face-mask therapy. Am J Orthod Dentofacial Orthop, St. Louis, v. 113, no. 3 p. 333-343, Mar. 1998.

2. BAIK, H. S. Clinical results of the maxillary protraction in Korean children. Am J Orthod Dentofacial Orthop, St. Louis, v. 108, no. 6, p. 583-592, Dec. 1995.

3. BAIK, H. S. et al. The treatment effects of Frankel functional regulator III in children with Class III malocclusions. Am J Orthod Dentofacial Orthop, St. Louis, v. 125, no. 3, p. 294-301, Mar. 2004.

4. BAUMRIND, S. et al. Quantitative analysis of the orthodontic and orthopedic effects of maxillary traction. Am $\mathbf{J}$ Orthod St. Louis, v. 84, no. 5, p. 384-398, Nov. 1983.

5. CAPELOZZA FILHO, L. et al. Effects of dental decompensation on the surgical treatment of mandibular prognathism. Int $\mathbf{J}$ Adult Orthodon Orthognath Surg, Philadelphia, v. 11, no. 2, p. $165-180,1996$

6. CHONG, Y.; IVE, J.; ÅRTUN, J. Changes following the use of protraction headgear for early correction of Class III malocclusion. Angle Orthod, Appleton, v. 66, no. 5, p. 351-362, 1996

7. CONTE, A.; CARANO, A.; SICILIANI, G. A new maxillary protractor. J Clin Orthod, Boulder, v. 31, no. 8, p. 523-530, Aug. 1997.
8. DELAIRE, J. Confection du masque orthopédique. Rev Stomat Chir Maxillofac, Paris, v. 72, no. 5, p. 579-582, July/Aug. 1971

9. DELAIRE, J. Maxillary development revisited: relevance to the orthopaedic treatment of Class III malocclusions. Eur J Orthod London, v. 19, no. 3, p. 289-311, June 1997.

10. DELLINGER, E. L. A preliminary study of anterior maxillary displacement. Am J Orthod, St. Louis, v. 63, n. 5, p. 509-516, May 1973.

11. GALLAGHER, R. W.; MIRANDA, F.; BUSCHANG, P. H. Maxillary protraction: treatment and posttreatment effects. Am J Orthod Dentofacial Orthop, St. Louis, v. 113, n. 6, p. 612-619, June 1998.

12. GÖYENÇ, Y.; ERSOY, S. The effect of a modified reverse headgear force applied with a facebow on the dentofacial structures. Eur J Orthod, London, v. 26, no. 1, p. 51-57, Feb. 2004.

13. HAAS, A. J. Palatal expansion: just the beginning of dentofacial orthopedics. Am J Orthod, St. Louis, v. 57, no. 3, p. 219-255, Mar. 1970.

14. HAAS, A. J. The treatment of maxillary deficiency by opening the midpalatal suture. Angle Orthod, Appleton, v. 35, no. 3, p. 200-217, July 1965 .

15. HICKHAM, J. H. Maxillary protraction therapy: diagnosis and treatment. J Clin Orthod, Boulder, v. 25, no. 2, p. 102-113, Feb. 1991.

16. ISHII, H. et al. Treatment effect of combined maxillary protraction and chincap appliance in severe skeletal Class III cases. Am J Orthod Dentofacial Orthop, St. Louis, v. 92, no. 4, p. 304-312, Oct. 1987. 
17. JACKSON, G. W. KOKICH, V. G.S SHAPIRO, P. A Experimental and postexperimental response to anteriorly directed extraora force in young Macaca nemestrina. Am J Orthod, St. Louis, v. 75 , no. 3, p. 318-333, Mar. 1979

18. KAMBARA, T. Dentofacial changes produced by extraoral foward force in the Macaca irus. Am J Orthod, St. Louis, v. 71 no. 3, p. 249-277, Mar. 1977

19. KAPUST, A. J. SINCLAIR, P. M. TURLEY, P. K. Cephalometric effects of face mask/expansion therapy in Class III children: a comparison of three ages groups. Am J Orthod Dentofacial Orthop, St. Louis, v. 113, no. 2, p. 204-212, Feb. 1998.

20. KILICCOGLU, H.; KIRLIC, Y. Profile changes in patients with class III malocclusions after Delaire mask therapy. Am J Orthod Dentofacial Orthop, St. Louis, v. 113, no. 4, p. 453-462, Apr. 1998.

21. KIM, J. H. et al. The effectiveness of protraction face mask therapy: a meta-analysis. Am J Orthod Dentofacial Orthop St. Louis, v. 115 , no. 6, p. 675-685, June 1999

22. MACEY-DARE, L. V. The early management of Class III malocclusions using protraction headgear. Dent Update, London, v. 27 no. 10 , p. $508-513$, Dec. 2000

23. McNAMARA JR., J. A. An orthopedic approach to the treatment of Class III malocclusion in young patients. J Clin Orthod, Boulder, v. 21, no. 9, p. 598-608, Sept. 1987

24. MERMIGOS, J.FULL, C. A. ANDREASEN, G Protraction of the maxillofacial complex. Am J Orthod Dentofacial Orthop St. Louis, v. 98, no. 1, p. 47-55, July 1990.

25. MIYASAKA-HIRAGA, J.; TANNE, K.; NAKAMURA, S. Finite element analysis for stresses in the craniofacial sutures produced by maxillary protraction forces applied at the upper canines. $\mathbf{B r} \mathbf{J}$ Orthod, London, v. 21, no. 4, p. 343-348, Nov. 1994.

26. NANDA, R. Protraction of maxilla in rhesus monkey by controlled extraoral forces. Am J Orthod, St. Louis, v. 74, no. 2 p. 121-141, Aug. 1978

27. NANDA, R.; HICKORY, W. Zygomaticomaxillary suture adaptations incident to anteriorly-directed forces in Rhesus monkeys. Angle Orthod, Appleton, v. 54, no. 3, p. 199-210, July 1984.

28. NARTALLO-TURLEY, P. E.; TURLEY, P. K. Cephalometric effects of combined palatal expansion and facemask therapy on Class II malocclusion. Angle Orthod, Appleton, v. 68, no. 3, p. 217-224 June 1998.

29. NGAN, P. W. et al. Treatment response and long-term dentofacial adaptations to maxillary expansion and protraction. Semin Orthod, Orlando, v. 3, no. 4, p. 255-264, Dec. 1997.

30. NGAN, P. et al. Treatment response to maxillary expansion and protraction. Eur J Orthod, London, v. 18, no. 2, p. 151-168, Apr. 1996.
31. SAADIA, M. TORRES, E. Sagittal changes after maxillary protraction with expansion in Class III patients in the primary, mixed and late mixed dentitions: a longitudinal retrospective study. Am J Orthod Dentofacial Orthop, St. Louis, v. 117, no. 6 669-680, June 2000

32. SANDIKÇIOGLU, M.; HAZAR, S. Skeletal and dental changes after maxillary expansion in mixed dentition. Am J Orthod Dentofacial Orthop, St. Louis, v. 111, no. 3, p. 321-327, Mar. 1997.

33. SHANKER, S. et al. Cephalometric: a point changes during and after maxillary protraction and expansion. Am J Orthod Dentofacial Orthop, St. Louis v. 110, no. 4, p. 423-430, Oct. 1996.

34. SILVA FILHO, O. et al. Intentional ankylosis of deciduous canines to reinforce maxillary protraction. J Clin Orthod, Boulder, v. 37 , no. 6, p. 315-320, June 2003.

35. SILVA FILHO, O. G.; MAGRO, A. C.; CAPELOZZA FILHO, L. Early treatment of the Class III malocclusion with rapid maxillary expansion and maxillary protraction. Am J Orthod Dentofacial Orthop, St. Louis, v. 113, no. 2, p. 196-203, Feb. 1998

36. SILVA FILHO, O. G.; MAGRO, A. C.; OZAWA, T. O. Má oclusão de Classe III: caracterização morfológica na infância (dentaduras decídua e mista). Ortodontia, São Paulo, v. 30, n. 2, p. 7-20, maio/ago. 1997.

37. SMITH, S. W.; ENGLISH, J. D. Orthodontic correction of a Class III malocclusion in an adolescent patient with a bonded RPE and protraction face mask. Am J Orthod Dentofacial Orthop St. Louis, v. 116, no. 2, p. 177-183, Aug. 1999

38. SUDA, N. et al. Effective treatment plan for maxillary protraction: Is the bone age useful to determine the treatment plan? Am J Orthod Dentofacial Orthop, St. Louis, v. 118, no. 1, P. 55-62, July 2000

39. SUNG, S. J.; BAIK, H. S. Assessment of skeletal and dental changes by maxillary protraction. Am J Orthod Dentofacial Orthop St. Louis, v. 114, no. 5, p. 492-502, Nov. 1998

40. TAKADA, K.; PETDACHAI, S.; SAKUDA, M. Changes in dentofacial morphology in skeletal Class III children treated by a modified maxillary protraction headgear and a chin cup: a longitudinal cephalometric appraisal. Eur J Orthod, London, v. 15, n. 3, 211-221, June 1993

41. TANNE, K.; SAKUDA, M. Biomechanical and clinical changes of the craniofacial complex from orthopedic maxillary protraction. Angle Orthod, Appleton, v. 61, no. 2, p. 145-152, Summer 1991.

42. TURLEY, P. K. Orthopedic correction of Class III malocclusion with palatal expansion and custom protraction headgear. J Clin Orthod, Boulder, v. 22, no. 5, p. 314-325, May 1988

43. WISTH, P. J. et al. The effect of maxillary protraction on the front occlusion and facial morphology. Acta Odontol Scand, Stockholm, v. 45, no. 3, p. 227-237, June 1987.
Endereço de correspondência

Omar Gabriel da Silva Filho

Rua Silvio Marchione, 3-20

CEP 17.012-900, Bauru/SP

E-mail: ortoface@travelnet.com.br 